\title{
SEXUAL IMPRINTING IN FEMALE ZEBRA FINCHES: CHANGES IN PREFERENCES AS AN EFFECT OF ADULT EXPERIENCE
}

\author{
by \\ SABINE OETTING and HANS-JOACHIM BISCHOF ${ }^{1\rangle}$ \\ (Lehrstuhl für Verhaltensforschung, Fakultät Biologie Universität Bielefeld, Postfach \\ 100131, 33501 Bielefeld, Germany)
}

(Acc. 18-X-1995)

\begin{abstract}
Summary
It has been shown that sexual imprinting in male zebra finches can be seen as a two stage process with an early acquisition period, where a sexual preference is established, and a consolidation process, where the early acquired preference is linked to sexual behaviour and stabilized. The consolidation process can, by the appropriate experimental design, be delayed until adulthood. In the course of consolidation, the initial preference for the foster species can be altered by exposure to a female of another species. Our results indicate that sexual imprinting in females is also a two stage process. In contrast to males, the consolidation period, at least under the experimental design used in this study, is longer than that obtained for males. It is speculated that the speed of consolidation could be enhanced by additional stimuli influencing female mate choice, as for example breeding facilities.
\end{abstract}

\section{Introduction}

A series of recent investigations (Bischof \& Clayton, 1991; Immelmann et al., 1991; Kruijt \& Meeuwissen, 1991, 1993; Oetting et al., 1995; for a review see Bischof, 1994) indicate that sexual imprinting in male zebra finches (Taeniopygia guttata castanotis) can be seen as a two stage process. According to this idea, sexual imprinting consists of two different stages,

\footnotetext{
1) We gratefully acknowledge the comments of two unknown referees. The study was supported by the Deutsche Forschungsgemeinschaft (Bi 245/9).
} 
an acquisition phase when the birds learn about their social environment (including their parents) and a consolidation process where the acquired information is linked to sexual behaviour and stabilized. Acquisition normally takes place while the birds are still dependent on their parents, and the consolidation process starts as soon as sexual behaviour occurs. Immelmann et al. (1991) and Kruijt \& Meeuwissen (1991) showed that male zebra finches, which had been reared by Bengalese finch (Lonchura striata) foster parents, and were isolated during adolescence consolidated their preferences for this species when first exposed as adults to a female Bengalese finch, even if the exposure was very short (Immelmann et al., 1991: $2 \times 20 \mathrm{~min}$; Kruijt et al., 1991: $3 \times 30 \mathrm{~min}$ ). Subsequent exposure to a female zebra finch did not alter the sexual preference any longer. By contrast, if the first exposure as an adult was to a female zebra finch, some of the males shifted their preferences towards zebra finches. Experiments of Bischof \& Clayton (1991) provided further evidence that the males' preferences can be changed only during the first exposure to a female. Oetting et al. (1995) provided additional details of the acquisition and the consolidation process. They demonstrated that modification of the previous preferences correlates with two factors: the amount of social information acquired at the acquisition period, and the level of arousal induced by the first exposure to a female or by another arousing situation. They also clearly showed that acquisition and consolidation are two independent processes.

The experiments which uncovered the two stage process in imprinting were as yet performed only with male zebra finches. There is ample evidence that female zebra finches also learn the features of their future mates by imprinting (e.g. Sonnemann \& Sjölander, 1977; Immelmann et al., 1978; Weismann et al., 1994; Vos, 1995). There are, however, indications that males and females may be differently affected by social experience, and that there may be a difference in imprintability between males and females (for a review see ten Cate, 1985).

In this study, we wanted to examine whether the idea of a two stage process in sexual imprinting may also apply to female zebra finches. Our results confirm that female zebra finches are sexually imprinted in two steps as well as males are, and they indicate that there are some differences in sexual imprinting, at least in the time course of the two steps. 


\section{Methods}

Figure 1 depicts the rearing and testing conditions. Eggs of zebra finch pairs were transferred to, and reared by, Bengalese finch foster parents, whose own eggs were removed. In each case, a whole clutch was transferred, and so the number of siblings varied between zero and four. Each breeding pair was housed in a separate cage $(80 \times 40 \times 30 \mathrm{~cm})$. At 40 days of age, the young birds were isolated in extra cages $(40 \times 30 \times 30 \mathrm{~cm})$ in another room where they could hear, but not see, other male and female zebra finches. Food and water were available ad libitum. Food consisted of dry and sprouted seed as well as occasional fresh greens. A multivitamin preparation was added to the water 2 or 3 times weekly. Pairs raising young also got a mixture of cooked egg, minerals and vitamins. The bird rooms received daylight and were supplementary lighted by artificial light, so that the animals received at least 14 hours of light. The temperature was held around $24^{\circ} \mathrm{C}$.

Thirty-one females were randomly distributed to three groups, which differed in the sequence of exposure to males after reaching 100 days of age (Fig. 1). Females of the $\mathrm{Bf} /$ - group were exposed to a male Bengalese finch, which was raised by zebra finch foster parents, for $1 \mathrm{~h}$ at 100 days of age. For exposure, the males were put into the home cage of the female. After 7 days of isolation the females were tested three times, once a day, on successive days, in a preference test lasting $20 \mathrm{~min}$ (test series A). In these tests, the females could choose between a male Bengalese finch and a male zebra finch, both reared by zebra finch parents. The test cage (Fig. 2) consisted of three compartments arranged in a line. The choice males were housed in the lateral compartments $(30 \times 40 \times 40 \mathrm{~cm})$, the test female in the centre compartment $(60 \times 40 \times 40 \mathrm{~cm})$. (For a detailed discription of the method used to determine a female's preference, see below.)

After 4 month of visual isolation, the females were tested again in a second test series (series B), which was designed identically to test series A. After 4 weeks of visual isolation,

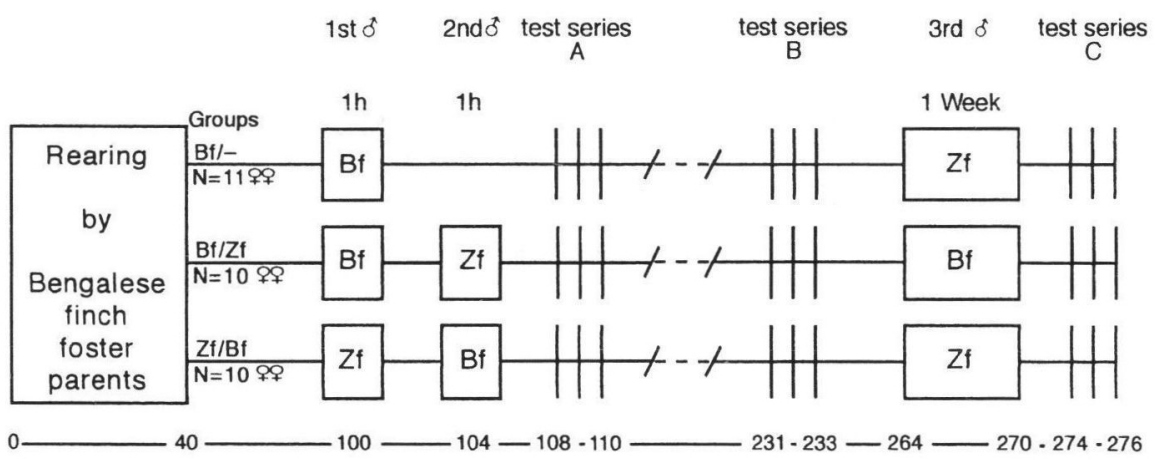

Days post hatching

Fig. 1. Outline of the experimental design of the three groups (Bf/-, Bf/Zf, Zf/Bf). Zf: Zebra finch, Bf: Bengalese finch. N: Number of females in each group. 


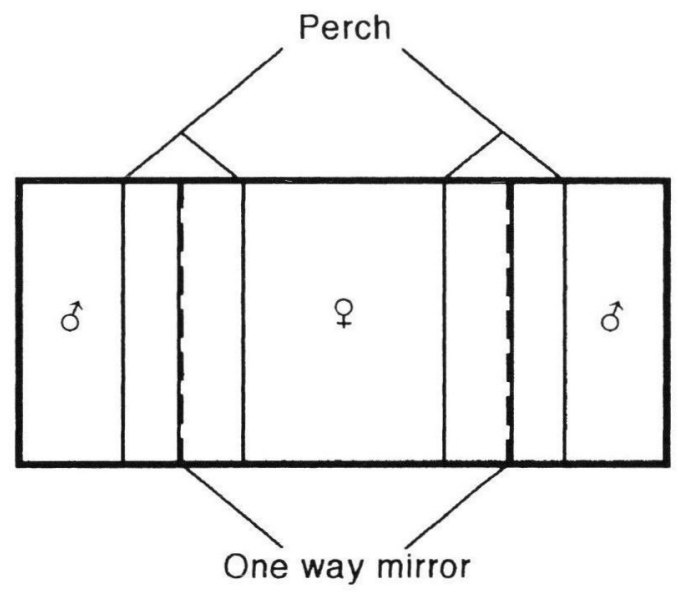

Fig. 2. Design of the test cage. Further explanation see text.

at the age of 264 days, the females of the Bf/- group were exposed to a male for 1 week, this time to a male zebra finch. After 3 days of isolation, the preference was determined in test series $\mathrm{C}$, which was also designed identical to test series $\mathrm{A}$ and $\mathrm{B}$.

Females of the Bf/Zf group were exposed to a male Bengalese finch for $1 \mathrm{~h}$ at 100 days of age. After 3 days of isolation, at the age of 104 days, the females were exposed for $1 \mathrm{~h}$ to a male zebra finch, at the same hour of the day as the first exposure. After 3 days of isolation, the preferences of these females were determined in test series $\mathrm{A}$, and test series $\mathrm{B}$ was performed after 4 month of isolation. After 4 weeks of isolation, at the age of 264 days, the females of this group were again exposed to a male of the same species as in the first exposure, this time for 1 week. After 3 additional days of isolation, the preference of the females was measured in test series $\mathrm{C}$ (Fig. 1).

The experimental design for females of the Zf/Bf group was identical to the females of $\mathrm{Bf} / \mathrm{Zf}$ group. The only difference was the order of the males presented. The females were first exposed to a male zebra finch at 100 days of age, and secondly exposed to a male Bengalese finch at 104 days of age. The third exposure to a male of the same species as the male of the first exposure at 264 days of age was, accordingly, to a male zebra finch (Fig. 1).

It has been shown that in addition to morphological features of a male its behaviour has an influence on the choice of a female (Bossema \& Kruijt, 1982; Kruijt et al., 1982; ten Cate \& Mug, 1984; Collins, 1994). Our priority in these experiments was to investigate the influence of the morphological features. We therefore tried to eliminate courtship behaviour of the test males as complete as possible. We separated the compartments by one way mirrors which allowed the females in the center compartment to see the test males, but the test males could not see the female. In addition, all tests were performed with the same two test males, which were selected on the basis of equal activity. These males 
were housed with a zebra finch female between tests. The test males were exchanged from test to test between the two outer compartments. During the tests, we never saw courtship behaviour displayed by the test males.

The centre compartment contained two perches, one each in front of the lateral compartments (Fig. 2). Every $10 \mathrm{~s}$ the position and orientation of the female was recorded. If the female was sitting at the perch or on the floor below the perch adjacent to and in front of a male (less the one body lenght), but was not facing it, this was counted as one. If the female was also facing the male, this gave an additional count. When the female was sitting at the perch or on the floor below the perch adjacent to the male and was facing it, from a distance of more than one body lenght, this also counted one. Consequently, a female could get 0,1 or 2 counts every $10 \mathrm{~s}$. A preference score, $\mathrm{Zf} /(\mathrm{Zf}+\mathrm{Bf})$, was calculated over the 3 tests in each series. In preference calculations, $\mathrm{Zf}$ and $\mathrm{Bf}$ equal the sums of scores for position in front of and orientation towards the male zebra finch and Bengalese finch, respectively. This score becomes zero if the two behaviours are exclusively shown to the male Bengalese finch, and 1.00 when the behaviours are exclusively directed to the male zebra finch. In addition, every tail quivering of a female towards a test male during the test situations was recorded. All observations were done by one of the authors, who was also involved in controlling the experimental time schedule. It was therefore not possible to take blind observations in all cases. But we are quite confident that a potential observer bias did not have an effect on our results. On the basis of previous results with males, we expected that the first exposure had a clear-cut influence on the consolidation of a female's preference, which is obviously not the case in the present experiments.

Tail quivering was not shown very frequently by the females. For those 22 (out of 264) tests where tail quivering was shown, an additional preference score was calculated according to the formula $\mathrm{PS}=\mathrm{Zf} /(\mathrm{Zf}+\mathrm{Bf})$. For this calculation $\mathrm{Zf}$ indicates the number of a female's tail quivering bouts towards the male zebra finch, and $\mathrm{Bf}$ indicates the number of a female's tail quivering bouts towards the male Bengalese finch. For the 22 cases where both scores could be determined, a Spearman rank correlation showed a highly significant relation between the two scores $(N=22$, Spearman's $r=0.55, p<0.01)$. Because tail quivering is a clear indicator for female sexual preferences, this confirms that the preference score based on position and orientation is also suitable for the determination of female sexual preferences. Therefore only these scores are shown in the following results.

\section{Statistics}

The overall effect of the rearing conditions of each group $(\mathrm{Bf} /-, \mathrm{Bf} / \mathrm{Zf}, \mathrm{Zf} / \mathrm{Bf})$ on the preferences in test series $\mathrm{A}, \mathrm{B}$ and $\mathrm{C}$ was evaluated by means of Friedman tests. If these tests yielded significant results $(p<0.05)$, the source of the difference was evaluated by a posthoc analysis according to Siegel \& Castellan (1988, p. 180). To test for statistical differences between the different rearing groups in each of the test series (A, B, C), Kruskal-Wallis tests were calculated. If these tests yielded significant results $(p<0.05)$, the significance of differences between groups was examined by means of a post-hoc analysis according to Siegel \& Castellan (1988, p. 213). All tests were two-tailed. 


\section{Results}

The open bars in Fig. 3 show the preference scores of the Bf/- group for test series $\mathrm{A}, \mathrm{B}$ and $\mathrm{C}$. One of the 11 females died before test series $\mathrm{C}$, so for this female results are only shown for test series $\mathrm{A}$ and $\mathrm{B}$. The medians (1st and 3rd quartile) in preference scores of the three test series were as follows: test series A: $0.29(0.27,0.32), N=11$; test series B: $0.35(0.26$, $0.45), N=11$; test series C: $0.28(0.17,0.43), N=10$. There were no significant differences between the preference scores of the three test series (Friedman test: $N=10, \chi^{2}=3.2, \mathrm{NS}$ ). In conclusion, the females of the $\mathrm{Bf} /$ - group developed a preference for male Bengalese finches, which was not altered substantially by 4 month of isolation, and was not influenced by the subsequent exposure to a male zebra finch.

Preference scores of the $\mathrm{Bf} / \mathrm{Zf}$ group for test series $\mathrm{A}, \mathrm{B}$ and $\mathrm{C}$ are depicted as dark bars in Fig. 3. One of the 10 females died after test series A. The medians (1st and 3rd quartile) in preference scores of the

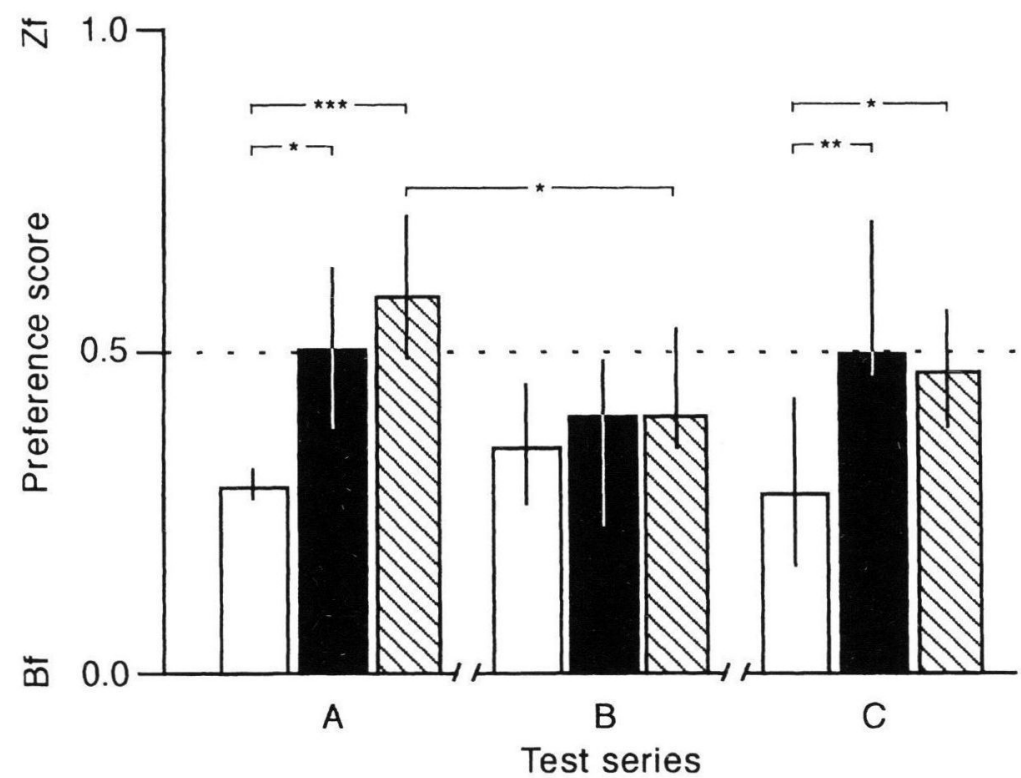

Fig. 3. The median, first and third quartile of the preference scores of the females in group Bf $/$ - (open bars), Bf/Zf (dark bars) and $\mathrm{Zf} / \mathrm{Bf}$ (hatched bars) in test series $\mathrm{A}, \mathrm{B}$ and $\mathrm{C}$.

$$
{ }^{*}=p<0.05,{ }^{* *}=p<0.01,{ }^{* * *}=p<0.001 \text {. }
$$


three test series were as follows: test series A: $0.50(0.37,0.63), N=10$; test series B: $0.39(0.22,0.48), N=9$; test series C: $0.49(0.45,0.7)$, $N=9$. The medians indicate that the females of the $\mathrm{Bf} / \mathrm{Zf}$ group did not develop a clear preference for male Bengalese or zebra finches, respectively. Remarkably, the preference scores of 7 out of 9 females decreased after 4 month of isolation (test series B) and increased towards a zebra finch preference again after the third exposure (before test series $\mathrm{C}$ ), although this exposure was to a male Bengalese finch. But, these changes in preferences were, although suspicious, not significant (Friedmann test: $N=9, \chi^{2}=$ $5.2, p<0.07$ ).

The hatched bars in Fig. 3 show the preference scores of the Zf/Bf group for test series A, B and C. One of the 10 females died before test series B and $C$. The medians (1st and 3rd quartile) in preference scores of the three test series were as follows: test series A: $0.59(0.49,0.72), N=10$; test series B: $0.4(0.35,0.54), N=9$; test series C: $0.47(0.38,0.57), N=9$. The medians of the scores again indicated no clear preferences for either species as well as in the $\mathrm{Bf} / \mathrm{Zf}$ group. Similar to the results of the $\mathrm{Bf} / \mathrm{Zf}$ group, all 9 females had lower preference scores after 4 month of isolation, and for 7 of the 9 females the preference scores increased again after the third exposure. In this case a Friedman test indicated significant differences between the preference scores of the three test series $(N=9$, $\chi^{2}=9.31, p<0.01$ ). A post-hoc comparison between the groups (Siegel \& Castellan, 1988, p. 180) showed a significant difference between the preference scores of test series A and B $(p<0.05)$. There was no significant difference between the preference scores of test series $\mathrm{A}$ and $\mathrm{C}$, and $\mathrm{B}$ and $\mathrm{C}$, respectively.

An analysis of the three groups $(\mathrm{Bf} /-, \mathrm{Bf} / \mathrm{Zf}, \mathrm{Zf} / \mathrm{Bf})$ concerning the preference scores of test series $A$ indicated significant differences (KruskalWallis test: $N=31, H=15.21, p=0.0005$ ). A post-hoc comparison between the groups (Siegel \& Castellan, 1988, p. 213) revealed significant differences which are depicted in Fig. 3. The preference scores of the $\mathrm{Bf} /$ - group (open bars) were significantly lower than the preference scores of the $\mathrm{Bf} / \mathrm{Zf}$ group (dark bars, $p<0.05$ ) and the preference scores of the $\mathrm{Zf} / \mathrm{Bf}$ group (hatched bars, $p<0.001$ ), respectively. There were no significant differences between the three groups after 4 month of isolation (test series B; Kruskal-Wallis test: $N=29, H=1.41$, NS). Similar 
differences as in test series A could be detected in test series $\mathrm{C}$ (KruskalWallis test: $N=28, H=11.47, p<0.01$, Fig. 3). The post-hoc comparison revealed significant differences between the preference scores of the $\mathrm{Bf} /-$ group and $\mathrm{Bf} / \mathrm{Zf}$ group $(p<0.01)$ and the $\mathrm{Bf} /-$ group and the $\mathrm{Zf} / \mathrm{Bf}$ group $(p<0.05)$.

\section{Discussion}

The results of the $\mathrm{Bf} /$ - group show that female zebra finches which were reared by Bengalese finch foster parents develop a sexual preference for male Bengalese finches when they were first exposed to a male Bengalese finch as adults and had no further exposure before the first test series. This matches with the results of earlier experiments with female zebra finches where an influence of the foster species on the development of sexual preferences was demonstrated (e.g. Sonnemann \& Sjölander, 1977; Immelmann et al., 1978; Vos, 1995). Our experiments also show that this preference remains stable after 4 months of isolation, and is not affected by a later exposure to a male zebra finch.

If, however, female zebra finches reared by Bengalese finch foster parents were exposed as adults to a male Bengalese finch and to a male zebra finch before the first test series $(\mathrm{Bf} / \mathrm{Zf}$ and $\mathrm{Zf} / \mathrm{Bf})$, their median preference scores expressed no clear preferences for either species, regardless whether the first exposure was to a male Bengalese finch and the second to a male zebra finch or vice versa. This indicates that in both groups the experience with a male zebra finch affects sexual preference. This is similar to results from experiments on sexual imprinting in male zebra finches, which were performed with a comparable design as described here (Bischof \& Clayton, 1991; Immelmann et al., 1991; Kruijt \& Meeuwissen, 1991, 1993; Oetting et al., 1995). In these studies, sexual experience with an unknown female after reaching adulthood could also change sexual preferences. But, in contrast to our results with females, male preferences were modified only, if the first sexual encounter was with a female of the unknown species. If the first exposure was to a female of the same species as the (foster) parents, a second exposure to a female of a different species did not change the sexual preference any more. As already mentioned in the introduction, this can be explained if the first exposure to a female has 
a consolidating effect on the neural representation which is acquired during rearing by the (foster) parents.

Our results thus indicate that one exposure to a male as an adult may not be sufficient for consolidation of female preferences, because the second exposure also had an effect on the preference. The third exposure before test series $\mathrm{C}$ obviously was not able to alter the acquired preference any longer. Although we cannot fully exclude that this is due to an age effect, we prefer to interpret this finding as to show that consolidation takes place in females as well as in males, but it is slower, at least in our experiments.

The experimental design we chose may be one reason for the prolongation of the consolidation process. Alatalo et al. (1986), Johnson \& Searcy (1993) and Hoi et al. (1994) have shown that the choice of a sexual partner in female birds depends not only on the phenotype of the male, but also on the quality of the territory and/or the nest. Therefore the females probably may be consolidated quicker if the male would be exposed in combination with, for example, breeding opportunities. This, however, has to be examined in further experiments.

The stability of the acquired preferences is one of the criteria defining imprinting (Immelmann \& Suomi, 1981). To test this feature, test series $\mathrm{B}$ and $\mathrm{C}$ were performed. There were no significant differences between test series $\mathrm{A}$ and $\mathrm{C}$ in all three experimental groups. This confirms that the preferences acquired as a consequence of the exposures during the acquisition and the consolidation period remain stable, even if there is a longer lasting exposure to another male in the meanwhile. Therefore the learning process observed here can be categorized as imprinting.

In one group $(\mathrm{Bf} / \mathrm{Zf})$, however, there was a significant difference between test series $\mathrm{A}$ and $\mathrm{B}$, respectively, in that the preference scores were lower (more directed to the rearing species) in series B. The same trend, although not significant, could be seen in the $\mathrm{Zf} / \mathrm{Bf}$ group. This shows that there is still some flexibility in the choice behaviour of the females. This, at the first glance, might be interpreted as to indicate that the one hour experience of the adult female with a male zebra finch had lost its influence on the preference of the female. In this case, the result would be contradictive to the two stage idea. However, in test series $\mathrm{C}$, the preference scores return to values similar to test series $\mathrm{A}$ irrespective of whether the third exposure (between tests B and C) were towards a male zebra finch 
(Zf/Bf group) or towards a male Bengalese finch (Bf/Zf group). Therefore we prefer to interpret the drop of the preference scores in test series $B$ as an effect of isolation. Isolation may affect the balance between the influence of the different representations which are developed in the course of the imprinting process (Bateson, 1981; Bischof, 1994; Oetting et al., 1995), but this effect is not permanent, and the previous balance is restored if the birds gain new social experience.

Taken together, our results confirm the claims of previous studies that female zebra finches can be sexually imprinted. They show that the two stage concept is applicable also in females. The differences between males and females is shown by the fact that only the first exposure to a female as an adult has an effect on the male's preference, while the female's preference is affected by more than one exposure to a male as an adult. We presume that the female needs additional information about the quality of the male to be stimulated adequately. Because these additional parameters are lacking in our experiments, the consolidation process may be slowed down compared to males.

\section{References}

Alatalo, R.V., Lundberg, A. \& Glynn, C. (1986). Female pied flycatchers choose territory quality and not male characteristics. - Nature 323, p. 152-153.

Bateson, P. (1981). The control of sensitivity to the environment during development. - In: Behavioral development (K. Immelmann, G.W. Barlow, L. Petrinovich \& M. Main, eds). Cambridge University Press, Cambridge, p. 432-453.

Bischof, H.-J. (1994). Sexual imprinting as a two-stage process. - In: Causal mechanisms of behavioural development (J.A. Hogan \& J.J. Bolhuis, eds). Cambridge University Press, Cambridge, p. 82-97.

- — \& Clayton, N. (1991). Stabilization of sexual preferences by sexual experience in male zebra finches Taeniopygia guttata castanotis. - Behaviour 118, p. 144-155.

Bossema, I. \& Kruijt, J.P. (1982). Male activity and female mate acceptance in the mallard (Anas platyrhynchos). - Behaviour 79, p. 313-324.

ten Cate, C. (1985). On sex differences in sexual imprinting. - Anim. Behav. 33, p. 13101317.

— \& Mug, G. (1984). The development of mate choice in zebra finch females. Behaviour 90, p. 125-150.

Collins, S.A. (1994). Male displays: cause or effect of female preference? - Anim. Behav. 48 , p. $371-375$.

Hoi, H., Schleicher, B. \& Valera, F. (1994). Female mate choice and nest desertion in penduline tits, Remiz pendulinus: the importance of nest quality. - Anim. Behav. 48, p. 743-746. 
Immelmann, K., Kalberlah, H.-H., Rausch, P. \& Stahnke, A. (1978). Sexuelle Prägung als möglicher Faktor innerartlicher Isolation beim Zebrafinken. - J. Orn. 119, p. 197-212.

- - Pröve, R., Lassek, R. \& Bischof, H.-J. (1991). Influence of adult courtship experience on the development of sexual preferences in zebra finch males. - Anim. Behav. 42, p. 83-90.

- — \& Suomi, S.J. (1981). Sensitive phases in development. - In: Behavioral development (K. Immelmann, G.W. Barlow, L. Petrinovich \& M. Main, eds). Cambridge University Press, Cambridge, p. 395-431.

Johnson, L.S. \& Searcy, W.A. (1993). Nest site quality, female mate choice, and polygyny in the house wren Troglodytes aedon. - Ethology 95, p. 265-277.

Kruijt, J.P., Bossema, I. \& Lammers, G.J. (1982). Effects of early experience and male activity on mate choice in mallard females (Anas platyrhynchos). - Behaviour 80, p. $32-43$.

- — \& Meeuwissen, G.B. (1991). Sexual preferences of male zebra finches: effects of early and adult experience. - Anim. Behav. 42, p. 91-102.

- $-\&-$ - (1993). Consolidation and modification of sexual preferences in adult male zebra finches. - Neth. J. Zool. 43, p. 68-79.

Oetting, S., Pröve, E. \& Bischof, H.-J. (1995). Sexual imprinting as a two-stage process: mechanisms of information storage and stabilization. - Anim. Behav. 50, p. 393-403.

Siegel, S. \& Castellan, N.J. (1988). Nonparametric statistics for the behavioural sciences. - McGraw Hill, New York.

Sonnemann, P. \& Sjölander, S. (1977). Effects of cross-fostering on the sexual imprinting of the female zebra finch Taeniopygia guttata. - Z. Tierpsychol. 45, p. 337-348.

Vos, D.R. (1995). Sexual imprinting in zebra finch females: do females develop a preference for males that look like their father? - Ethology 99, p. 252-262.

Weisman, R., Shackleton, S., Ratcliffe, L., Weary, D. \& Boag, P. (1994). Sexual preferences of female zebra finches: imprinting on beak colour. - Behaviour 128, p. 15-24. 